\title{
Frustration effects in magnetic molecules
}

\author{
Jürgen Schnack \\ Universität Osnabrück, Fachbereich Physik, D-49069 Osnabrück, Germany
}

\begin{abstract}
Besides being a fascinating class of new materials, magnetic molecules provide the opportunity to study concepts of condensed matter physics in zero dimensions. This contribution will exemplify the impact of molecular magnetism on concepts of frustrated spin systems. We will discuss spin rings and the unexpected rules that govern their low-energy behavior. Rotational bands, which are experimentally observed in various molecular magnets, provide a useful, simplified framework for characterizing the energy spectrum, but there are also deviations thereof with far-reaching consequences. It will be shown that localized independent magnons on certain frustrated spin systems lead to giant magnetization jumps, a new macroscopic quantum effect. In addition a frustration-induced metamagnetic phase transitions will be discussed, which demonstrates that hysteresis can exist without anisotropy. Finally, it is demonstrated that frustrated magnetic molecules could give rise to an enhanced magnetocaloric effect.
\end{abstract}

PACS numbers: 75.50.Xx,75.10.Jm,75.40.Cx

\section{INTRODUCTION}

Geometric frustration of interacting spin systems is the driving force of a variety of fascinating phenomena in low-dimensional magnetism ${ }^{11}$ In this context the term frustration describes a situation where in the ground state of a classical spin system not all interactions can be saturated simultaneously. A typical picture for such a situation is a triangle of antiferromagnetically coupled spins, where classically the spins are not in the typical up-down-up configuration, but assume a ground state that is characterized by a relative angle of $120^{\circ}$ between neighboring spins. This special classical ground state characterizes several frustrated spin systems, among them giant Keplerate molecules, 2 the triangular lattice antiferromagnet, and the kagome lattice antiferromagnet. 


\section{J. Schnack}

Throughout the article the spin systems are modeled by an isotropic Heisenberg Hamiltonian augmented with a Zeeman term, i.e.,

$$
\underset{\sim}{H}=-\sum_{u, v} J_{u v} \underset{\sim}{\vec{s}}(u) \cdot \underset{\sim}{\vec{s}}(v)+g \mu_{B} B \underset{\sim}{S_{z}}
$$

$\underset{\sim}{\vec{S}}(u)$ are the individual spin operators at sites $u, \underset{\sim}{\vec{S}}$ is the total spin operator, and $\underset{\sim}{S_{z}}$ its $z$-component along the homogeneous magnetic field axis. $J_{u v}$ are the matrix elements of the symmetric coupling matrix. In the following we will consider only antiferromagnetic couplings which are characterized by a negative value of $J_{u v}$.

\section{GENERALIZED SIGN RULE FOR SPIN RINGS}

Rigorous results on spin systems such as the sign rule of Marshall and Peierls ${ }^{3}$ and the famous theorems of Lieb, Schultz, and Mattis ${ }^{45}$ have sharpened our understanding of magnetic phenomena. With the advent of finite size antiferromagnetic spin rings the question arose whether such general statements can also be made for odd spin rings which are not decomposable into two sublattices, i.e. not bipartite. A key quantity of interest is the shift quantum number $k=0, \ldots N-1$ associated with the cyclic shift symmetry of the rings. The corresponding crystal momentum is then $2 \pi k / N$. For rings with even $N$ (bipartite) one can explain the shift quantum numbers for the relative ground states in subspaces $\mathcal{H}(M)$ of total magnetic quantum number $M ![345$ In recent investigations we could numerically verify, that even for frustrated rings with odd $N$ astonishing regularities hold. Unifying the picture for even and odd $N$, we find for the ground state without exception! ${ }^{6}$ The ground state belongs to the subspace $\mathcal{H}(S)$ with the smallest possible total spin quantum number $S$. If $N \cdot s$ is integer, then the ground state is non-degenerate. If $N \cdot s$ is half integer, then the ground state is fourfold degenerate.

The sign rule of Marshall and Peierls can be generalized for all subspaces $\mathcal{H}(M)$ with a given total magnetic quantum number!

$$
\text { If } N \neq 3 \quad \text { then } \quad k \equiv \pm a\left\lceil\frac{N}{2}\right\rceil \bmod N, \quad a=N s-M .
$$

Moreover the degeneracy of the relative ground state is minimal. Here $\lceil N / 2\rceil$ denotes the smallest integer greater than or equal to $N / 2$. "Minimal degeneracy" means that the relative ground state in $\mathcal{H}(M)$ is twofold degenerate if there are two different shift quantum numbers and non-degenerate if $k=0$ $\bmod N$ or $k=N / 2 \bmod N$, the latter for even $N$. 


\section{Frustration effects in magnetic molecules}

The k-rule (2) is founded in a mathematically rigorous way for $N$ even, ${ }^{345} N=3, a=0, a=1$, and in part for $a=2$ [ For the ground state with $N$ odd and $s=1 / 2$ the k-rule follows from the Bethe ansatz ${ }^{[7}$ An asymptotic proof can be formulated for large enough $N$ for systems with an asymptotically finite excitation gap (Haldane systems) $\stackrel{6}{[}$ The k-rule also holds for the exactly solvable $X Y$-model with $s=1 / 2$. For $N \cdot s$ being half integer field theory methods yield that the ground state shift quantum number approaches $N / 4$ for large $N{ }^{[8}$ Apart from these findings a rigorous proof of the k-rule still remains a challenge.

\section{ROTATIONAL MODES AND MAGNETIZATION JUMPS}
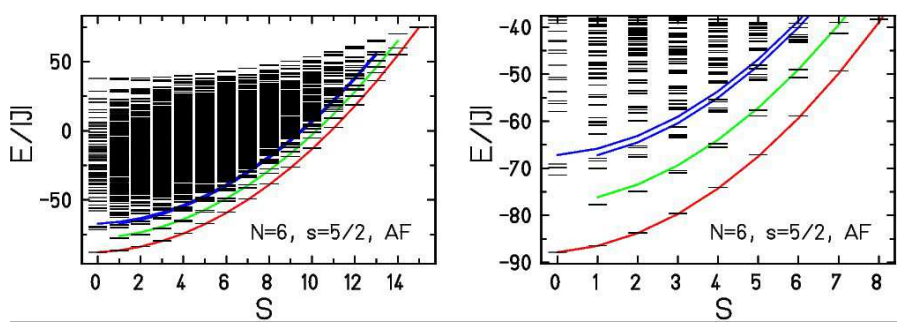

Fig. 1. Energy spectrum (1.h.s.) of a ring of six antiferromagnetically coupled spins $s=5 / 2$ and close-up view of the low-energy part (r.h.s.). The lowest rotational bands are highlighted by solid curves.

An antiferromagnet that can be decomposed into two sublattices has as its lowest excitations the rotation of the Néel vector as well as spin wave excitations. ${ }^{[9}$ In finite size systems these excitations are arranged in rotational (parabolic) bands as shown in Fig. 1 for a ring of six antiferromagnetically coupled spins $s=5 / 2$. Such a behavior is most pronounced for bipartite, i.e. unfrustrated systems. 1011

Contrary to this behavior the minimal energies of certain frustrated antiferromagnetic molecules of cuboctahedral and icosidodecahedral structure depend linearly on total spin $S$ above a certain total spin. ${ }^{12}$ Such a dependence, which is depicted on the l.h.s. of Fig. 2. results in an unusually big jump to saturation as can be seen on the r.h.s. of Fig. 2 Although first noticed for the Keplerate molecule $\left\{\mathrm{Mo}_{72} \mathrm{Fe}_{30}\right\}$, such a behavior is quite common for a certain class of frustrated spin systems such as the kagome or the pyrochlore lattice. 1314 The underlying reason is that due to the special geometric frustration in such systems - polygons are surrounded by triangles - the relative ground states in subspaces $\mathcal{H}(M)$ are for big enough $M$ given 


\section{J. Schnack}
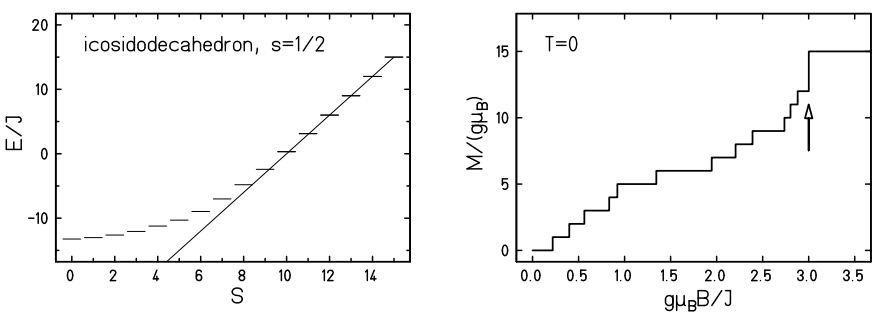

Fig. 2. L.h.s.: Minimal energies of the icosidodecahedron for $s=1 / 2$. The highest four levels fall on a straight line. R.h.s.: Resulting $(T=0)$ magnetization curve. The magnetization jump of $\Delta M=3$ is marked by an arrow.

by product states of independent localized magnons. Therefore, the energy scales linearly with the number of independent magnons which in turn is linearly related to $M$ or $S \underline{13}$

\section{HYSTERESIS WITHOUT ANISOTROPY}
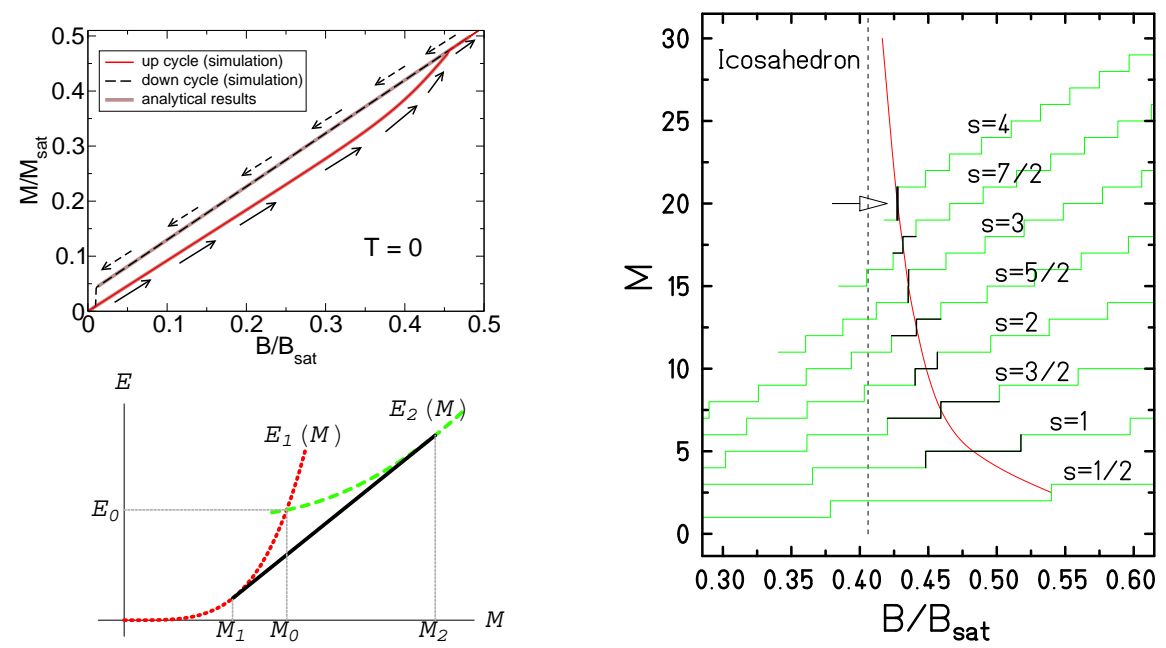

Fig. 3. L.h.s. (top): Hysteresis loop of the classical icosahedron. L.h.s. (bottom): Minimal energy curves of the classical icosahedron. R.h.s.: Magnetization curves of the quantum icosahedron for various $s$ at $(T=0) ! \frac{15}{[}$

The observation of hysteresis effects in magnetic materials is usually an outcome of their magnetic anisotropy. In a recent article we could report 


\section{Frustration effects in magnetic molecules}

that magnetic hysteresis (Fig. 3 1.h.s., top) occurs in a spin system without any anisotropy 15 Specifically, we investigated an icosahedron where classical spins mounted on the vertices are coupled by antiferromagnetic isotropic nearest-neighbor Heisenberg interaction giving rise to geometric frustration. At $T=0$ this system undergoes a first order metamagnetic phase transition at a critical field $B_{\mathrm{c}}$ between two distinct families of ground state configurations. The metastable phase of the system is characterized by a temperature and field dependent survival probability distribution. Our exact classical treatment shows that the abrupt transition at $T=0$ originates in the intersection of two energy curves belonging to different families of spin configurations that are ground states below and above the critical field (Fig. 3 l.h.s., bottom). The minimum of the two energy functions constitutes a non-convex minimal energy function of the spin system and this gives rise to a metamagnetic phase transition. We could also show that the corresponding quantum spin system for sufficiently large spin quantum number $s$ possesses a non-convex set of lowest energy levels when plotted versus total spin. Fig. 3 (r.h.s.) shows the $(T=0)$-magnetization curves for various $s$. The magnetization plateaus of smallest width are highlighted on each curve. At $s=4$ a magnetization jump of $\Delta M=2$ occurs, marked by the arrow. At $s=3$ a tiny plateau persists. The solid curve shows that the field values that bisect the smallest plateaus converge to the classical transition field (dashed line).

\section{ENHANCES MAGNETOCALORIC EFFECT}

The magnetocaloric effect, which consists in cooling or heating of a magnetic system in a varying magnetic field, can assume especially large values if the entropy $S(T, B)$ changes drastically as a function of field according to

$$
\left(\frac{\partial T}{\partial B}\right)_{S}=-T \frac{\left(\frac{\partial S}{\partial B}\right)_{T}}{C(T, B)} .
$$

This can for instance happen at phase transitions. In the context of frustrated spin systems huge cooling rates (3) can be achieved for certain topologies in the vicinity of the saturation field due to the large (sometimes even macroscopic) degeneracy of independent magnon states. 16 17/18

Fig. 4 shows the isentropes of a cuboctahedron with $s=1 / 2$ (l.h.s.) as well as a spin ring with $N=12$ spins $s=1 / 2$ (r.h.s.). The cuboctahedron hosts independent magnons and thus features an unusually big magnetization jump to saturation whereas the spin ring does not. Consequently the isentropes of the cuboctahedron have a steeper slope above the saturation field, therefore the cuboctahedron exhibits a larger cooling rate. 


\section{J. Schnack}
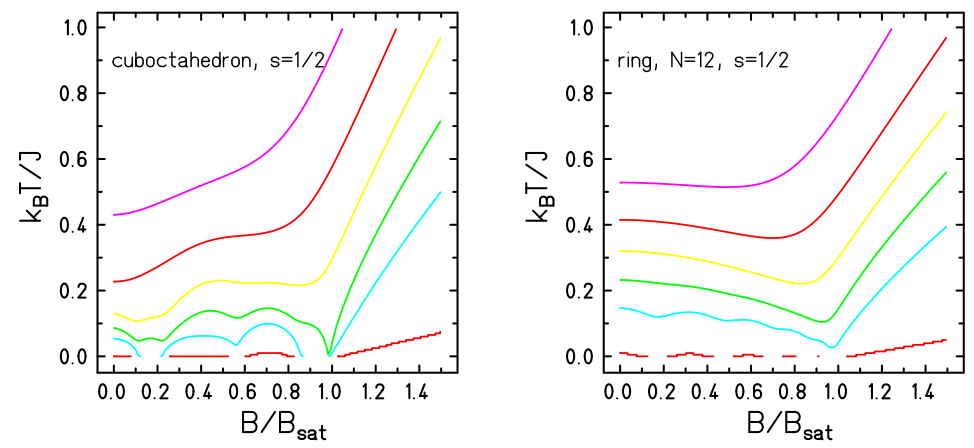

Fig. 4. Isentropes of the cuboctahedron with $s=1 / 2$ (l.h.s.) as well as a spin ring with $N=12$ spins $s=1 / 2$ (r.h.s.).

\section{ACKNOWLEDGMENTS}

It is my pleasure to thank K. Bärwinkel, A. Honecker, P. Kögerler, M. Luban, J. Richter, H.-J. Schmidt, and C. Schröder for the fruitful collaboration that produced so many exciting results.

\section{REFERENCES}

1. J. Greedan, J. Mater. Chem. 11, 37 (2001).

2. A. Müller et al., Chem. Phys. Chem. 2, 517 (2001).

3. W. Marshall, Proc. Royal. Soc. A (London) 232, 48 (1955).

4. E. H. Lieb, T. Schultz, and D. C. Mattis, Ann. Phys. (N.Y.) 16, 407 (1961).

5. E. H. Lieb and D. C. Mattis, J. Math. Phys. 3, 749 (1962).

6. K. Bärwinkel, P. Hage, H.-J. Schmidt, and J. Schnack, Phys. Rev. B 68, 054422 (2003).

7. M. Karbach, Ph.D. thesis, Bergische Universität - Gesamthochschule Wuppertal, 1994.

8. I. Affleck, D. Gepner, H. Schulz, and T. Ziman, J. Phys. A 22, 511 (1989).

9. P. W. Anderson, Phys. Rev. 86, 694 (1952).

10. J. Schnack and M. Luban, Phys. Rev. B 63, 014418 (2001).

11. O. Waldmann et al., Phys. Rev. Lett. 91, 237202 (2003).

12. J. Schnack, H.-J. Schmidt, J. Richter, and J. Schulenburg, Eur. Phys. J. B 24, 475 (2001).

13. J. Schulenburg et al., Phys. Rev. Lett. 88, 167207 (2002).

14. J. Richter et al., J. Phys.: Condens. Matter 16, S779 (2004).

15. C. Schröder et al., Phys. Rev. Lett. 94, 207203 (2005).

16. M. E. Zhitomirsky and A. Honecker, J. Stat. Mech.: Theor. Exp. 2004, P07012 (2004).

17. O. Derzhko and J. Richter, Phys. Rev. B 70, 104415 (2004).

18. J. Schnack, R. Schmidt, and J. Richter, in preparation. 